\title{
Periodontal treatment during pregnancy did not reduce the occurrence of poor pregnancy outcomes
}

\author{
Abstracted from \\ Offenbacher S, Beck JD, Jared HL, et al. \\ Effects of periodontal therapy on rate of preterm delivery: a randomized controlled trial. \\ Obstet Gynecol 2009; 114: 551-559 \\ Address for correspondence: Steven Offenbacher, North Carolina Oral Health Institute, Durham NC 27709, \\ USA. E-mail: Steve_Offenbacher@dentistry.unc.edu
}

\section{Question: Does the treatment of periodontal disease during pregnancy reduce the incidence of preterm birth?}

\footnotetext{
Design This was a randomised controlled trial (RCT).
} Intervention Participants were assigned randomly to receive periodontal treatment either before 23 weeks' gestational age or after delivery. Treatment consisted of up to four sessions of supragingival and subgingival scaling and root planing using hand and ultrasonic instruments to complete the baseline therapy. Local anaesthesia was used as needed. Those in the treatment group also received full-mouth tooth polishing and oral hygiene home instructions. There were no followup periodontal treatment visits. After delivery, those in the delayed-treatment group received periodontal therapy at no cost. Outcome measure The principal outcome (gestational age $<37$ weeks) included induced or spontaneous deliveries, foetal demise and miscarriage, but not therapeutic abortions. Secondary outcomes included gestational age $<35$ weeks; mean birth weight among neonates adjusted for race, sex and gestational age; and a composite measure of neonatal morbidity and mortality before discharge. . Results The trial randomised 1806 patients at three performance sites and 1760 evaluable patients completed the study. At baseline, there were no differences between the treatment and control arms for any of the periodontal or obstetric measures. The rate of preterm delivery for the treatment group was $13.1 \%$, and $11.5 \%$ for the control group (P 0.316). There were no significant differences when comparing women in the treatment group with those in the control group with regard to the adverse event rate or the major obstetric and neonatal outcomes.

Conclusions Periodontal therapy did not reduce the incidence of preterm delivery.

\section{Commentary}

This is a large $(\mathrm{N}=1760)$, well-designed RCT that aimed to assess the effects of nonsurgical periodontal therapy on poor pregnancy outcomes. The primary outcome of the study was the proportion of births with a gestational age of $<37$ weeks. Secondary outcomes included: incidence of gestational age $<35$ weeks; mean birth weight among neonates adjusted for race, sex and gestational age; and a composite measure of neonatal morbidity and mortality before discharge defined as: foetal demise after randomisation; neonatal death before discharge from hospital to home or chronic care; respiratory distress syndrome; proven sepsis; grade 3 or 4 intraventricular haemorrhage; or necrotising enterocolitis requiring treatment.

Patients were enrolled at five healthcare centres, and a random permutation that stratified participants by clinical centre was used. The groups were appropriately balanced at baseline and the subjects assigned to the control group received periodontal treatment after delivery. Inclusion/ exclusion criteria as well as criteria for periodontal exposure were explicitly stated, dental examiners were masked to treatment assignment until after the primary obstetric outcome was collected, and the values for intra- and inter-examiner reliability of periodontal recordings were considerably high.

Pregnancies that did not end in a live birth were regarded as part of the unfavourable outcome and the analyses were in general conducted using the intention-to-treat principle.

This study addressed clear and focused questions; the major pertinent, clinical outcomes were considered; and compliance was very good throughout the study. All patients who were eligible and randomised were properly accounted for at the end of the study.

Periodontal treatment during pregnancy resulted in a statistically significant improvement in the levels of probing depth, clinical attachment and bleeding on probing, but there were no significant differences between the treatment and the control groups with regard to the major outcomes investigated. Periodontal therapy during pregnancy did not affect the occurrence of preterm birth, low birth weight or pre-eclampsia. These results confirm the findings of a well-designed and properly conducted multicentre RCT on the topic, which did not find any effect of periodontal therapy before 21 weeks on the same outcomes. ${ }^{1}$

Practice points

- Nonsurgical periodontal therapy before 23 weeks gestational age seems not to reduce the occurrence of poor pregnancy outcomes.

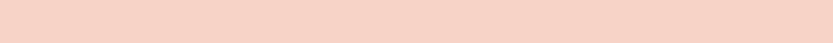

Rodrigo López

Department of Periodontology, Faculty of Health Sciences, University of Aarhus, Aarhus, Denmark

1. Michalowicz BS, Hodges JS, DiAngelis AJ, et al. Treatment of periodontal disease and the risk of preterm birth. N Engl / Med 2006; 355: 1885-1894.

Evidence-Based Dentistry (2009) 10, 105. doi:10.1038/sj.ebd.6400681 